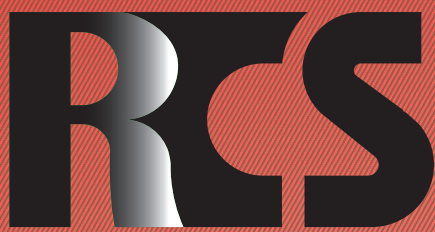

Depósito legal ppi $201502 Z U 4662$

Esta publicación científica en formato digital es continuidad de la revista impresa Depósito Legal: pp $197402 Z$ U789

- ISSN: 1315-9518 • ISSN-E: 2477-9431

Revista de Ciencias Sociales

Universidad del Zulia. Revista de la Facultad de Ciencias Económicas y Sociales Vol. XXVII,

No. 4,2021

Esta publicación científica en formato digital es continuidad de la revista impresa Depósito Legal: pp $197402 Z U 789$ ISSN: $1315-9518$ 


\title{
Habilidades del Trabajador(a) Social: Desde la mirada de su acción profesional
}

\author{
Guillén de Romero, Jacqueline Coromoto*
}

\begin{abstract}
Resumen
El profesional de Trabajo social tiene dentro de sus funciones contribuir con el proceso de transformación para el beneficio de las personas que conviven en una comunidad, lo cual implica contar con habilidades específicas que coadyuven a su efectiva labor, por ello, la necesidad de determinar las habilidades del Trabajador(a) Social, desde la mirada de su acción profesional. Se asumieron los aportes de De la Rosa (2017); Espinoza y Ramón (2020); Guillen et al. (2021); entre otros. Se desarrolló bajo el paradigma positivista, un estudio descriptivo, transversal, de campo, encuestando una población representativa de profesionales de Trabajo Social de Venezuela y Ecuador, aplicando la encuesta con cuestionario con escala de Likert vía online. El análisis de los datos fue con distribución frecuencial. Los resultados indicaron que casi siempre los encuestados manifiestan sus habilidades personales, cognitivas, metodológicas y sociales que describen su ser, saber, hacer e interactuar. Se concluye, que en casi todas las áreas en las cuales se desenvuelve, demuestra sus capacidades asumiendo como importante intervenir y mediar, de allí que, las habilidades cognitivas o técnicas propician sus conocimientos para actuar, investigar, sistematizar, brindando apoyo a las personas en sus situaciones bien sean individuales, grupales o comunitarias, demostrando habilidades personales.
\end{abstract}

Palabras clave: Habilidades; trabajador(a) social; acción profesional; Venezuela; Ecuador.

Post-Doctora en Gerencia de la Educación Superior. PhD. en Ciencias Jurídicas. MgSc. en Intervención Social. Licenciada en Trabajo Social. Abogada. Profesora de la Universidad Técnica de Manabí, Portoviejo, Ecuador. E-mail: jacqueline.guillen@utm.edu.ec (iD) ORCID: https://orcid.org/0000-0002$\underline{9112-1910}$

Recibido: 2021-06-16

- Aceptado: 2021-08-31 


\title{
Skills of the Social Worker: From the perspective of their professional action
}

\begin{abstract}
The social work professional has within its functions to contribute to the transformation process for the benefit of the people who live together in a community, which implies having specific skills that contribute to their effective work, therefore, the need to determine the skills of the Social Worker, from the perspective of their professional action. The contributions of De la Rosa (2017) were assumed; Espinoza and Ramón (2020); Guillen et al. (2021); among others. A descriptive, cross-sectional, field study was developed under the positivist paradigm, surveying a representative population of Social Work professionals from Venezuela and Ecuador, applying the survey with a Likert scale questionnaire online. The data analysis was with frequency distribution. The results indicated that almost always the respondents manifest their personal, cognitive, methodological and social skills that describe their being, knowing, doing and interacting. It is concluded that in almost all the areas in which it operates, it demonstrates its capabilities assuming it is important to intervene and mediate, hence, cognitive or technical skills propitiate its knowledge to act, investigate, systematize, providing support to people in their situations, whether individual, group or community, demonstrating personal skills.
\end{abstract}

Keywords: Skills; social worker; professional action; Venezuela; Ecuador.

\section{Introducción}

Los tiempos de cambios que caracterizan la sociedad del siglo XXI, ha incidido en todos los ámbitos de la vida tanto personal como profesional, al tomar en cuenta que el ser humano debe adaptar sus conocimientos y actuaciones a las exigencias sociales, por lo cual, en el aspecto profesional, tanto las carreras como los perfiles de egreso de los estudiantes deben ser cónsonos con los requerimientos operativos, por cuanto, es necesario formar para la acción. En ese sentido, se considera que la carrera de Trabajo Social, ha tenido que modificar muchos aspectos tanto teóricos como prácticos, para que el profesional pueda cumplir con los propósitos para lo cual fue conformada esta disciplina, considerando importante conocer cuáles habilidades necesita para poder llevar a cabo la acción social.

Por lo cual, la acción del Trabajo Social (TS) se orienta a la práctica y a la intervención en la realidad social, de allí que el profesional debe asumir al hombre, a las comunidades, sus necesidades, expectativas sociales, desde la perspectiva individual, grupal como también colectiva, convirtiéndose esta finalidad en su razón de ser, saber, hacer, para interactuar con ellos con la finalidad de poder cumplir con su propósito que es favorecer tanto el bienestar como la calidad de los individuos en su contexto social.

Por tal razón, se destaca que la acción social del Trabajo Social, es la intervención, vista como algo más que una práctica profesional, por lo cual "a través de las acciones en las que se propone y ejecuta se articulan principios éticos y de control social que pocas veces son advertidos, tenidos en cuenta o cuestionados" (Moreno y Molina, 2018, p.1); considerando de allí, que los propósitos que se persiguen son relevantes y necesarios dada su orientación al cambio en las relaciones sociales, al mejoramiento en la calidad de vida, a la mitigación al sufrimiento por diversas causas, o a la atención a condiciones de vulnerabilidad.

En función de lo expuesto, el objeto de 
la acción social es aquello de lo que se ocupa el Trabajo Social, al intervenir al individuo $\mathrm{y}$ al entorno, atendiendo tanto a cuestiones relacionadas con las condiciones materiales, como la alimentación; o inmateriales, como lo es la seguridad, destacando que no solo se interviene en problemas, también se hace en capacidades y oportunidades. En cuanto al objeto, existe cierto consenso que en la acción social el profesional no debe, en su afán de resolver los problemas, olvidarse de las personas, por lo cual, el propósito de los trabajadores(as) sociales es lograr el bienestar social, la calidad de vida, aquello que tiene que ver con la autonomía individual y la integración social, tal como lo explica De la Rosa (2017).

Por ello, los agentes que se implican en la acción, así como los que se sienten afectados, los profesionales, específicamente en este caso, los(las) trabajadores(as) sociales requieren de habilidades para colocarlas en práctica en su intervención. Esto, favorece su relación con la gente, les da credibilidad al brindarle el apoyo y la orientación necesaria para lograr los cambios pertinentes de manera individual, grupal o comunitaria.

Asimismo, "el entorno socio- familiar, es un punto clave, ya que buena parte de los problemas sociales y el entorno, tienen que ver en la causa y en la solución de los mismos" (De la Rosa, 2017, p.7); asi como el organizativo, por cuanto la acción social habitualmente se realiza en el marco de una organización, la cual, con sus valores, misiones, prioridades, estilo de trabajo, verá condicionada de manera relevante la actividad del trabajador(a) social.

El entorno social, también debe constituirse en objeto importante, así como los voluntarios quienes juegan un papel fundamental. Son personas que por diferentes motivos invierten su tiempo en colaborar en programas de Trabajo Social. Esta colaboración debe hacerse siempre en el marco de un programa social, técnicamente bien definido y supervisado por un profesional. La responsabilidad de los sujetos, se refiere al papel que los destinatarios deben jugar en los procesos y asumir en la determinación de los objetivos, procesos y esfuerzos para el cambio.

Por consiguiente, la acción social involucra al sujeto, agente, actor como destinatarios de la intervención sujetos activos del cambio. Son ellos, los que deciden cambiar qué, para qué y cómo. Aunque frecuentemente los condicionantes sociales sitúan a las personas en un mero papel de agentes que desarrollan conductas previsibles, normalizadas, la acción de intervención debe contribuir a promocionar el papel de actor, de sujetos conscientes y libres. Por lo tanto, la acción social, se observa como una unidad desde las intenciones hasta las relaciones y acciones con los ciudadanos. En ese orden de ideas, Fantova (2018) menciona que:

Parece evidente la identificación del objeto de la intervención social y de las necesidades y situaciones que se encontrarían dentro de su perímetro de actuación y el avance del conocimiento en la construcción de conceptos e instrumentos que permitan aproximarse a dichos fenómenos. (p.10)

Tal posición, se plantea porque el profesional debe asumir también esos estados no tan evidentes, para los que se utilizan expresiones como riesgo, fragilidad o vulnerabilidad y su modificación; por lo cual, resulta crítico intensificar la capacidad preventiva de la acción social, como lo son, las habilidades con las que cuenta para poderse acercar, orientar, prevenir, corregir, intervenir lo que está fuera de orden, y necesita del apoyo, de la guía para mejorar los aspectos personales, grupales o comunitarios, debiendo utilizar técnicas de estratificación o segmentación para la diversificación de itinerarios, para la intervención social hoy y aquí.

Ahora bien, es pertinente recordar que el(la) profesional de Trabajo Social, para cumplir con los propósitos de la acción social, debe tener unas habilidades importantes caracterizadas por su perfil, y con las cuales puede cumplir con sus funciones y roles, asi como es necesario contar con ciertas estrategias metodológicas que coadyuven al desarrollo de las actividades, lo cual implica que teniendo estos elementos podría garantizar el éxito de su práctica con la persona, el grupo y la comunidad. En tal sentido, se plantea la 
interrogante ¿Cuáles habilidades debe poseer el(la) profesional de Trabajo Social, para cumplir con la acción social?, proponiendo como objetivo: Determinar las habilidades del Trabajador(a) Social desde la mirada de su acción profesional.

\section{Fundamentación teórica}

\subsection{Perfil profesional del Trabajador Social}

Para entender la acción social desarrollada por el(la) profesional de Trabajo Social, es importante tomar en cuenta el perfil personal y profesional que debe tener, de allí llegar a sus habilidades personales, cognitivas, metodológicas, emocionales, sociales, comunitarias, que constituyen el ser, saber, hacer e interactuar para lograr su acción social en casos individuales, de grupos o de comunidad, partiendo de su perfil profesional.

El perfil del profesional de Trabajo Social en la Universidad del Zulia (Maracaibo, Venezuela), tiene un proceso de formación enmarcado en "el modelo de currículo integral propuesto por Walter Peñaloza y adaptado comprendiendo las áreas curriculares: Formación General, Formación Profesional Básica, Formación Profesional Específica, Prácticas Profesionales, Orientación, Autodesarrollo y Servicio Comunitario. Para el diseño y ejecución del currículo integral" (Vargas-Acosta et al., 2016, p.84), donde el perfil académico profesional se fundamente en competencias, vinculadas a este profesional:

Las que definen los aspectos más particulares del ejercicio profesional, a saber: Promoción del Desarrollo Humano y de las Comunidades, Evaluación de Políticas Sociales, Terapia Social con Personas, Pequeños Grupos y Familias y Gerencia de Procesos, Proyectos y Programas Sociales. De allí que el egresado de la ETS-LUZ se percibe como un profesional experto en la promoción e intervención de las interacciones entre personas, familias, grupos, comunidades y organizaciones sociales. (p.84)
Por tanto, la Universidad del Zulia, además, de otras de Venezuela, asume un perfil integral donde se prepara al profesional de TS de manera que pueda cumplir con sus funciones de prevención, orientación, mediación, intervención, buscando la transformación individual, grupal o comunitaria de las personas que atiende. De igual manera, la Universidad Técnica de Manabí (2020), en Ecuador, plantea que:

La carrera de Trabajo Social forma profesionales técnicos e investigativos, de conciencia crítica, analítica y reflexiva, con capacidades múltiples que respondan a la demanda social y al buen desarrollo de sus competencias en favor del desarrollo del país y del mundo, sustentados en la interacción con individuos, grupos, familias y comunidades, empleando conocimientos científicos y metodológicos apropiados al campo de las Ciencias Sociales y del Comportamiento, que permita potencializar las capacidades humanas y ciudadanas, generando procesos de participación, inclusión y equidad, con el fin de lograr justicia y desarrollo humano en sus diversas manifestaciones. (p.2)

En ese orden de ideas, en esta universidad mencionada, así como en otras del país Ecuador, se pretende que un profesional de Trabajo Social, sea una persona con habilidades para aplicar una comunicación efectiva, tratando de encontrar soluciones a los problemas y conflictos que surgen entre las personas, quienes, con la ayuda pertinente, resuelvan enmarcados en la normativa legal, desarrollando entre todos, entendimiento, cooperación, cambio, para propender al bienestar individual y común.

Por eso, las funciones del trabajador social "consisten en promover una mejor calidad de vida y bienestar social a los diferentes sectores de la población en los que el profesional se involucre, gestionar y promocionar los recursos existentes entre usuarios y otros profesionales del contexto social" (Rodríguez, Calderón y Bravo, 2019, p.43), de forma tal que pueda contribuir con la transformación, practicando en su acción sus habilidades personales, técnicas, sociales. 


\subsection{Habilidades personales, el ser del profesional para la acción social}

Ser Trabajador(a) Social (TS), es algo muy importante, por cuanto se convierte en un profesional al servicio de los demás, su acción es para el bienestar de los otros, bien sea de manera individual, grupal o comunitaria, lo cual implica tener que estar interactuando con otras personas, indicando necesario poseer unas habilidades características de la personalidad que constituyen el ser, propiciando posibilidades de escuchar, acercarse, comprender, tener paciencia y tolerancia; por ello, podría decirse que no todas las personas pueden ejercer esta profesión.

Se parte del hecho que las habilidades personales son actitudes, comportamientos con los cuales el profesional puede realizar su labor, por cuanto "las personas desarrollan actitudes y comportamientos propios hacia los demás, desempeñan roles diferentes en sus vidas, toman decisiones cotidianamente, buscan defender sus puntos de vista" (Guillén et al., 2021, p.87), pudiendo asumir distintas posiciones de poder. En este proceso se valen de habilidades comunicacionales (Navarro et al., 2020), de capacidad para persuadir y negociar con la gente en búsqueda de soluciones satisfactorias para todos.

Para el logro de estas acciones sociales, es necesario asumir que: "Las habilidades y actitudes del TS están en su capacidad de relación con los usuarios y las técnicas son un medio para la acción que usa según sus fines, objetivos y necesidades" (Arias et al, 2013, p.43), entonces el(la) trabajador(a) social debe ser portador(a) de cualidades y valores, por ello, Ander-Egg (1986) menciona como habilidades personales:

La mística y vocación de servicio, porque aquel con quien trabaja no en un "cliente" sino una persona, ha de ser su preocupación central. En su práctica ha de reconocer el destino propio de cada individuo cuya dignidad trasciende toda consideración económica y toda contingencia histórica. (p.85)

Además, el autor antes citado, considera necesario tener madurez emocional, con la cual, un(a) trabajador(a) social actúa siempre con realidades humanas donde la cooperación, así como el conflicto se entremezclan permanentemente, frente a estas circunstancias, su control emocional juega un papel fundamental en cuanto a la capacidad de actuar equilibradamente con espíritu sereno y quieto, cuando se está bajo diferentes tipos de presiones.

En la actualidad, según lo menciona Henríquez (2017), se hace énfasis en el desarrollo de habilidades personales dentro del contexto de la inteligencia emocional, por el interés que el profesional demuestre fortalezas en cuanto a su autoconocimiento, autoestima, autocontrol, adaptación al cambio, manifieste empatía, adecuadas relaciones interpersonales, para llevar a cabo efectivo trabajo en equipo, resolución de problemas, liderazgo; además, habilidades organizacionales para gestionar recursos, capacidades de información, con iniciativa, espíritu emprendedor.

\subsection{Habilidades cognitivas, el saber para la acción social}

En relación, a las habilidades cognitivas, se pretende que el profesional de Trabajo Social pueda manifestar el desarrollo de procesos intelectuales como la memoria, atención, toma de decisiones y creatividad, así como las instrumentales con conocimientos computacionales y dominio de idiomas. La labor del trabajador(a) social, requiere de la adquisición de un complejo sistema de competencias durante su formación, que se logra durante la educación universitaria, mediante aspectos pedagógicos tales como los señalados por Espinoza y Ramón (2020):

Precisión y correspondencia entre el modelo del profesional, los contenidos de las disciplinas y los modos de actuación. Sistematización del tratamiento de las competencias profesionales desde un enfoque trans disciplinar. Trabajo metodológico integrado desde la carrera y las disciplinas. Práctica laboral, con énfasis en la investigación social para la solución de los problemas del contexto. Evaluación 
de las competencias considerando los conocimientos, las actitudes, los valores propios del desempeño y los modos de actuación. (p.316)

Aunado a las anteriores habilidades, el profesional de Trabajo Social, en su saber debe manejar normas y leyes, así como tener conocimiento de las distintas áreas de trabajo donde puede ejercer su acción social como lo son la familiar, escolar, judicial, de salud, comunitaria; de manera que su labor sea efectiva y garantice bienestar con el servicio que presta a los individuos, grupos y comunidad.

Además, ha de poseer competencias generales que demuestren: Compromiso ético, crítico, autocrítico y responsable; aprendizaje y aplicación de los conocimientos en la práctica; habilidad para tomar decisiones, de adaptación a nuevas situaciones; comprensión y expresión oral, así como escrita, con dominio del lenguaje especializado de la disciplina; mencionando la Universidad de Barcelona (2017), habilidades para buscar, usar e integrar la información con el empleo de las tecnologías de la información y las comunicaciones; valoración del impacto social y medio-ambiental de actuaciones en el ámbito propio; actitudes abiertas y empáticas basadas en el respeto, como también el reconocimiento ante la diversidad y la multiculturalidad; participación activa con espíritu emprendedor, al igual que de liderazgo.

Para Miranda et al. (2020), los TS identifican relevante la formación multidisciplinaria, por cuanto este saber, es coherente con los desafíos que los propios trabajadores sociales mencionan, al tener que intervenir en procesos de gestión de redes, control de gestión, así como en actividades de derecho, economía, sindicalismo, protección social, aspectos que demostrará cuando realice sus intervenciones sociales, sus visitas domiciliarias, las entrevistas sociales, cualquier otra metodología que le ayude a generar cambios en las personas, grupos y comunidades.

Las habilidades cognitivas, se refieren a los conocimientos técnicos que el profesional de Trabajo Social debe tener para desarrollar su acción social, es decir, manejar conocimientos de la actividad profesional, de las bases culturales, filosóficas, técnicas y científicas de dicha profesión, que permitirán asumir los hechos de la realidad, analizándolos a la luz de las teorías sociales, psicológicas, tomándose en cuenta factores que intervienen en los hechos que debe intervenir.

Estas habilidades cognitivas y técnicas, se adquieren en el desarrollo de la profesión, durante la formación en el ámbito universitario, como primera entrada, y luego se van fortaleciendo en la práctica, con adiestramiento continuo mediante talleres, cursos, diplomados, lo cual le permitirá al profesional de Trabajo Social adquirir los basamentos necesarios para hacer su diagnóstico, la planificación y ejecución de esas acciones, mediante el proceso de intervención y mediación.

\subsection{Habilidades metodológicas para el hacer en la acción social}

Elhacerdelprofesionalde Trabajo Social, implica el uso de metodologías específicas, conociéndose la intervención, la mediación, la investigación, como técnicas y procesos con los cuales se logran los objetivos establecidos en la acción social, considerándose que en su hacer lo primero que hace es el diagnóstico de la realidad estudiada, del entorno familiar, social, escolar, comunitario, de salud, jurídico, según sea su ámbito específico, sin obviar el manejo de teorías y modelos que son aspectos ampliamente analizados durante su formación académica.

En ese sentido, "la práctica del profesional se ordena a partir de cuatro momentos de reflexión metodológico: definición del problema objeto de intervención, selección de alternativas de acción, ejecución de actividades y evaluación" (Arias et al., 2013, p.43), estos momentos son integrados e incluyen otros procedimientos según los objetivos propuestos. Esta perspectiva ubica a la investigación, como un medio para definir el objeto de intervención, y a la 
planificación, como una ayuda para organizar en forma anticipada la acción; entonces, la investigación y planificación se estructuran como dos herramientas centrales de la acción profesional.

Resaltando los planteamientos más relevantes de los últimos tiempos en metodología del Trabajo Social, la intervención profesional "es la reconstrucción histórica del campo problemático lo cual indica hacer una lectura epistemológica del sobre qué, para qué, cómo y con quién se desarrolla el ejercicio profesional" (Rozas, 1998, p.35). Este campo problemático reproduce la relación funcional entre capital trabajo y protección social, transitando por las posibilidades, así como limitaciones que este proceso le permite; para encarar la relación, demanda recurso al articular la acción específica del TS con el contexto que consiente una reflexión dialéctica y crítica sobre las situaciones problemáticas que se establecen para la intervención profesional, lo cual devela la complejidad de la cuestión social.

Además, metodológicamente la intervención social implica que el profesional de TS, da acompañamiento y orientación a la persona en su proceso de autoayuda, considerando su protagonismo en el cambio, por lo cual, su función es ser facilitador y gestor, valiéndose de métodos, procedimientos, técnicas e instrumentos como motor impulsor de dicha transformación, es decir, contribuye a forjar la autonomía individual, colectiva y comunitaria, como lo expresan GarcíaDomingo y Sotomayor-Morales (2017), dejando de lado la antigua concepción de ayuda.

De igual manera, es relevante considerar desde el punto de vista metodológico percibir el entorno, a las personas, los conflictos, por tanto "para lograr este objetivo, es necesario cultivar las capacidades de investigación, así como integrar las teorías y métodos de conocimiento en proyectos de innovación, aplicando enfoques cualitativos y cuantitativos" (Suárez et al., 2020, p.336), contribuyendo esto a desarrollar un adecuado diagnóstico de la realidad a intervenir, asi como a asumir las estrategias de acción pertinentes para propiciar las transformaciones necesarias en cada caso.

En ese marco de ideas, dentro de las habilidades del profesional de Trabajo Social, se encuentra la práctica de las metodologías participativas de investigación y acción social con su fuerte propuesta epistemológica de investigar para la acción, para y con sus actores sociales protagonistas de lo que se intenta hacer desde lo participativo, generando estrategias en su quehacer con la gente y las comunidades.

\subsection{Habilidades sociales del profesional en su interacción con los otros}

Todo profesional de Trabajo Social, para direccionar su acción social, debe tener habilidades para interactuar con los demás, quién goza de esa capacidad de influir en los otros, tener carisma, para despertar en los demás respeto y autoridad, visto no como poder, sino como atracción, porque en su trabajo de casos individuales, de grupos y comunitarios, es necesario manejar las relaciones interpersonales, y sobre todo, saber comunicarse con las personas a quienes les brinda el servicio asistencial, al intervenir, así como mediar, haciendo muestra de sus habilidades comunicativas, como lo explica Barreto-Pico (2017), con el fin de comprender instrucciones verbales y escritas:

$\mathrm{Si}$ el trabajador social es quien conoce cómo se relacionan las personas, cómo trabajar con dichas relaciones y cómo funcionan las estructuras sobre las que se pretende actuar y en las que hay que buscar los recursos necesarios, evidentemente requiere de habilidades relacionadas con la comunicación oral y escrita. (p.481)

Por consiguiente, esto le permitirá escribir textos con distintos propósitos e interpretar información de cuadros y gráficos, analizar problemas y sus posibles soluciones, son el punto de partida para que las personas puedan aprender de manera continua y realizar diferentes actividades en los ámbitos: Personal, 
laboral, cultural y social, especialmente al mediar situaciones, problemas, así como conflictos.

El trabajo de mediar no siempre es posible hacerlo con efectividad, por lo cual, se requiere de unas características o condiciones personales y profesionales que debe adquirir; en este caso, el profesional de Trabajo Social como mediador, establece una relación de ayuda con la gente de la comunidad, para contribuir a la solución de los problemas y conflictos que puedan darse entre ellos, y por ello, tienen como objetivo acompañar a las partes en conflicto.

Ante estas premisas, el saber guiar la técnica de la mediación "facilita el restablecimiento de la comunicación, las relaciones interpersonales, limar asperezas entre las partes en disputa, quienes quedan satisfechas por los resultados obtenidos" (Guillén et al. 2021, p.87), planteando que a diferencia de aquellos que acuden a un juzgado donde colocan a las partes en actitud de adversario, las terceras partes actuantes intervienen antes que el proceso madure, dándole solución a todo o a nada, $\mathrm{y}$ muchas veces la comunicaciones se agravan en lugar de mejorar. La idea es que haya ganadores al encontrar alternativas óptimas para las partes, aumentando el protagonismo porque los actores son responsables de sus actos. Al respecto, Munuera y Minguela (2019), indican que el profesional de TS como mediador comunitario debe:

(...) trabajar en pro de los intereses comunes dirigidos hacia:

- Ayudar a la comunidad a tomar decisiones rápidas, consensuadas y comprometidas, a partir del respeto a sus valores y su historia.

- Alcanzar un compromiso ético de las personas que forman la comunidad.

- Repartir el poder y la responsabilidad desde la equidad, descentralizando la toma de decisiones.

- Ampliar el tamaño de la comunidad cuando sea necesario, para evitar la existencia de perjuicios. (p.55)

En este contexto, el(la) trabajador(a) social en su acción social mediadora puede ser visto como un modelo referente a seguir, entonces, si la mediación tiene un componente educativo, el mediador es el medio a través del cual se realiza este aprendizaje de las partes, por ello, el mediador tiene la responsabilidad de acercar los principios mediadores a su propia vida, para hacer congruente la enseñanza que, de un modo directo o indirectamente, realiza en el marco del desarrollo de la profesión. En ese sentido, Novel (2013) manifiesta que:

El mediador, establece una relación de ayuda, en la que el objetivo consiste en acompañar a las partes en conflicto, a:

- Identificar sus necesidades, intereses, dificultades y recursos, para desbloquear la situación de enrocamiento que genera el conflicto.

- Mejorar su modo de comunicación para poder expresarse de manera pacífica y resolutiva, facilitando el diálogo con las otras partes.

- Acercar posiciones entre las personas implicadas en el conflicto, para poder comprender, empatizar y gestionar el conflicto en un modo más funcional.

- Hacerse co-responsables acerca de la situación que están viviendo y así poder actuar de modo colaborativo

- Aprender a utilizar un sistema de comunicación no violento que les ayude a clarificar y resolver el conflicto presente y otros futuros. (p.2)

De igual manera, el mediador social, debe tener iniciativa personal combinada de modo complementario, con la iniciativa grupal, conocimientos amplios de mediación y disposición a aprender de modo continuado, así como visión amplia y enfocada hacia el futuro, al tiempo que comprensión profunda de la situación presente. Las cualidades mediadoras integradas en sí mismo, actúan como modelo y referente positivo, deben asumir capacidad de análisis, introspección, validación y evaluación sistemática, con habilidades comunicativas y relacionales, que faciliten aprendizaje mutuo.

Además, tener capacidad para soportar la presión y el estrés que genera la incertidumbre como las situaciones inesperadas, poseer creatividad permanente, amplio sentido del humor, para enfocar las múltiples situaciones que se dan en las organizaciones con 
imaginación para visualizar modos creativos de diseño, planificación e intervenciones diseñadas a medida.

También implica, tener capacidad de adaptación y afrontamiento positivo al cambio, de modo flexible y efectivo, con experiencia amplia, disposición positiva al reto de avanzar en nuevas direcciones, visión apreciativa sistemática e interiorizada, aportando una visión externa motivadora, capacidad de comunicar, motivar, dialogar, reconducir, reproducir de modo reformulado ideas, así como tener disposición a la reflexión, al aprendizaje continuo, para mejorar las prácticas.

Incluso, Novel (2013) indica que el TS debe tener capacidad de abstracción para relacionar, innovar, deducir y ampliar modos de intervención eficaces, capacidad para motivar a personas, equipos y grupos al cambio, así como a la mejora, tanto personal como colectiva, capacidad de compromiso con si mismo y con los demás, enfocando su visión hacia el éxito de los proyectos, asi como capacidad de trabajo en equipo multidisciplinar es fundamental, al igual que pensamiento estratégico, reflexivo y creativo, que posibilite la creatividad unida a la eficiencia.

Entonces, como ya se ha dicho, dentro de las habilidades sociales es importante en el TS, la comunicación como lo plantean Miranda et al. (2020), que implica considerar que el cómo se piensa influye en el cómo se habla y se comporta. Por ello, es imprescindible que el mediador realice los cambios internos necesarios para vivir los principios mediadores, para después transmitirlos en su vida cotidiana y profesional.

De acuerdo a la posición de Novel (2013), en mediación, la autenticidad y la transparencia, son elementos incluidos por sistema tanto en el comportamiento interno (pensamientos y sentimientos), como en el externo (lenguaje y comportamiento); y esto supone de un lado, reformular internamente los propios condicionantes, valores y creencias, así como de otra parte, significa colocar un especial énfasis en hablar y transmitir las ideas, necesidades, directrices a las personas del equipo, con enfoque pacífico y apreciativo. Esta transmisión debe ser fiel al ir acompañado de una serie de formas congruentes con lo que se quiere comunicar $\mathrm{y}$, sobre todo, ser facilitadores del diálogo.

En el mismo hilo conductual de la investigación, se trae a colación lo planteado por Barreto-Pico (2017), quien recuerda que es fundamental, el lenguaje que usa el facilitador o mediador en un proceso de gestión de las diferencias, el cual, debe contener y mostrar de modo claro los valores, así como principios de la mediación. De igual forma, se cuenta con lo expresado por los autores Munuera y Mínguela (2019); así como Miranda et al. (2020), quienes plantean importante que la comunicación que se establezca maneje de manera adecuada el tono, el volumen, la cadencia, el ritmo del habla, los contrastes y el tipo de voz; puesto que es un factor a tener en cuenta para comunicar serenidad, aceptación, tolerancia $\mathrm{o}$, por el contrario, ansiedad, incomodidad, nerviosismos, prisas.

Algunos aspectos básicos que deben ser tomados en cuenta, siguiendo la posición de Novel (2013), respecto a la comunicación no verbal, se resumen a continuación:

a. El silencio, el cual tiene siempre un significado y así debe ser valorado. Puede significar que la persona necesita un espacio para reflexionar sobre el tema, tener en cuenta los propios sentimientos, aunque a veces, simplemente, el silencio puede servir para tomarse unos segundos de respiro y también, debe identificarse su significado.

b. La mirada, porque mediante ella se puede evaluar el estado de comodidad o incomodidad del otro, pero también se puede transmitir seguridad, interés y animar a seguir con la conversación. Así mismo, a través de esta, se expresa el interés en el otro y en la relación. Es un tipo de anclaje que debe ser utilizado como tal en el marco de la comunicación efectiva.

c. El movimiento y la mímica, porque a través de ellos se pueden identificar estados emocionales o disposicionales, que el mediador debe reconocer y valorar, con el fin de que puedan ser utilizados a favor de una 
comunicación más eficaz.

d. Las distancias, pueden influir en la calidad y cantidad de comunicación que las partes intercambien con el mediador. Es importante saber utilizar este modo de contactar con el otro, evitando la sobreexposición, o, por el contrario, la minimización de la distancia que produciría barreras en la comunicación.

e. Las posiciones físicas, las cuales se refieren al lugar que ocupan las partes en el medio de interacción donde se encuentran, importantes para asegurar una buena comunicación; por lo que, se debe estar atento a que las partes presentes en la sesión tengan un buen contacto visual para fomentar la seguridad, la confianza, estén situadas a la misma altura para evitar desequilibrios, favoreciendo la utilización óptima de los modos de contacto y comunicación deseados, con posiciones confortables, con el fin de evitar que la incomodidad física, así como la fatiga obstaculicen la calidad del intercambio al provocar la interrupción prematura del encuentro, manejando las formas paralingüísticas y otros modos de comunicación no verbal.

Los aspectos mencionados se utilizan para invitar a las partes a continuar (a través de miradas, silencios acompañados y otras manifestaciones como señales con la cabeza, tacto y expresiones de $\mathrm{mmm}$, ajá) o, por el contrario, para limitar a las partes en su expresión verbal o evitar interrupciones en el relato de una de las partes (con signos con la mano, expresiones faciales o incluso el uso del tacto, que debe ser meticulosamente valorado antes, para evitar una reacción de rechazo) (Novel, 2013)

Además, se asume que la vestimenta es importante, por lo cual el(la) Trabajador(a) social debe tener en cuenta no solo cuestiones acerca del emisor, el mensaje, el canal o el contexto, sino también del receptor. La primera impresión es importante y las posteriores también. En un contexto determinado, la vestimenta y forma de presentarse deberá ser de un modo concreto; mientras que, en otro, será de otra naturaleza. El profesional de TS que trabaja en organizaciones, debe ser consciente que su imagen le antecede y si desea producir confianza e impacto positivo, deberá ser un aspecto sobre el que convendrá colocar un cuidado estratégico, como lo menciona Barreto-Pico (2017).

\section{Metodología}

Este estudio realizado con los lineamientos del paradigma positivista, fue descriptivo, transversal, de campo, totalizándose cincuenta y cuatro (54) profesionales de Trabajo Social, aunque al momento de recoger la información se consideró como población un grupo representativo de veinte (20) profesionales, escogidos con muestreo no probabilístico intencional, específicamente dieciséis (16) de la Universidad del Zulia, en Maracaibo, estado Zulia, Venezuela; y cuatro (4), de la Universidad Técnica de Manabí, Portoviejo, Ecuador; cuya selección se asumió con tres criterios: Ser docente de las universidades mencionadas, tener más de cinco (5) años de experiencia y estar dispuesto a participar en el estudio.

Con base en lo anterior, se seleccionaron $\mathrm{y}$ fueron encuestados con un cuestionario vía online (tomando en cuenta que por la pandemia el contacto presencial era muy difícil). Se elaboró para ello, un cuestionario conformado por veinte 20 preguntas (5 por cada tipo de habilidades), con escala de respuesta tipo Likert, siendo siempre, casi siempre, algunas veces, casi nunca y nunca, que fueron codificadas con $5,4,3,2$ y 1 punto, respectivamente. Se realizó a través del programa SPSS versión 23.0 el análisis estadístico, específicamente la distribución frecuencial, cuyos resultados permitieron determinar las habilidades del profesional de Trabajo Social, según, la percepción del grupo representativo de sujetos de esta disciplina. 


\section{Resultados y discusión}

Al procesar los datos aportados por los profesionales de Trabajo Social que participaron en este estudio, se conformó la Tabla 1, que determina cómo es según la opinión de esta población muestral, el comportamiento de las habilidades que manifiestan en su acción social.

\section{Tabla 1}

Habilidades del profesional de Trabajo social

\begin{tabular}{cccccc}
\hline Indicadores & Siempre & $\begin{array}{c}\text { Casi } \\
\text { siempre }\end{array}$ & $\begin{array}{c}\text { Algunas } \\
\text { veces }\end{array}$ & $\begin{array}{c}\text { Casi } \\
\text { nunca }\end{array}$ & Nunca \\
\hline Personales & 13,8 & 31,2 & 29,9 & 18,2 & 6,9 \\
Cognitivas & 19,9 & 31,9 & 27,9 & 17,7 & 2,6 \\
Metodológicas & 19,5 & 35,5 & 24,7 & 20,3 & 0,0 \\
Sociales & 17,7 & 32,9 & 27,7 & 18,5 & 3,16 \\
Promedio & $17,73 \%$ & $32,87 \%$ & $27,55 \%$ & $18,67 \%$ & $3,16 \%$ \\
\hline
\end{tabular}

Fuente: Elaboración propia, 2021.

Al observar los resultados puede considerarse que los profesionales de Trabajo Social participantes en este estudio, determinan que casi siempre según el 32,87\% de ellos, manifiestan en su acción social las habilidades personales, cognitivas o técnicas, metodológicas y sociales; el 27,55\% asume que algunas veces muestra estas habilidades mencionadas; para el 18,67\% casi nunca es así; mientras un $17,73 \%$ siempre las manifiesta; y el 3,16\% nunca lo hace.

Destaca, que estas habilidades se manifiestan casi siempre en todos los profesionales de Trabajo social, lo cual indica que de acuerdo al ámbito donde desarrolla su acción social, muestra unas más que otras como lo explica De la Rosa (2017), quién considera que para intervenir o mediar, el profesional debe conocer las características del objeto, tomando en cuenta que cualquier problema va a exigirle manifestar habilidades tanto personales como sociales y metodológicas.

De allí, que el TS demuestra estar muy relacionado con la cultura, los valores sociales y la política social porque su dirección de acción es la de asegurar condiciones de bienestar que hagan posible el ejercicio pleno de la libertad, por lo que deben considerarse de manera equilibrada los mecanismos de acceso y de arraigo para que la acción social sea inclusiva enmarcando dos formas de asumirla, en la administración del bienestar, y la segunda, en la ciudadanía activa.

Al observar los valores de la Tabla 1, el $35,5 \%$ de los encuestados respondieron que casi siempre muestran en su acción social las habilidades metodológicas, importantes para poder hacer sus actividades de intervención, mediación en el espacio donde le corresponde, generándose actividades de investigación acción participativa en los grupos y comunidades donde se desenvuelven profesionalmente.

Este resultado concuerda con el postulado de Galarza (2017), para quienes el Trabajo Social en el contexto actual latinoamericano pasa a ser una disciplina científica cuya herramienta de investigación posibilita alcanzar el desarrollo individual, así como colectivo, y el respeto a los derechos humanos, desarrollando la intervención y la mediación como un gestor activo de cohesión 
social; de allí, que su metodología de acción transformadora está encaminada al bienestar de los seres humanos en sociedad para obtener un mejor desarrollo humano, una vida digna, igualdad, equidad y la justicia social, por lo cual se entiende la importancia de su labor.

De igual manera, el $32,9 \%$ de los profesionales encuestados (ver Tabla 1) refirieron que casi siempre asumen las habilidades sociales para interactuar con las personas, los grupos y comunidades, tomando en cuenta la comunicación como el elemento clave para poder llegar a ellos, entenderlos durante la intervención o mediación que desarrolla, posibilitándole su acción social, y el logro de los propósitos determinados en cada caso.

Estos valores coinciden con los planteamientos de Miranda et al. (2020), quienes consideran la comunicación fundamental para interactuar con las personas, grupos y comunidades en momentos para intervenir y mediar, haciendo adecuado uso del tono de voz, el volumen, la cadencia, el ritmo del habla, los contrastes y el tipo de voz, que es un factor a tener en cuenta para comunicar serenidad, aceptación, tolerancia o, por el contrario, ansiedad, incomodidad, nerviosismos, prisas. Además, de adecuar la mirada, la sonrisa, la mímica, la postura, como elementos para saber acercarse a ellos de manera efectiva durante su acción social, manteniendo la distancia con respeto.

Asimismo, para el $31,9 \%$ de los encuestados, las habilidades cognitivas $\mathrm{o}$ técnicas casi siempre están presentes en su acción social, por cuanto se refiere a sus conocimientos pertinentes a la disciplina, a las técnicas y herramientas que le permiten acercarse e intervenir los casos individuales, grupales y comunitarios, tomando en cuenta lo que se quiere hacer y cómo lograrlo, de allí la importancia de saber hacerlo.

Esto concuerda con la teoría mencionada por Ramón et al. (2019), quienes expresan que el(la) Trabajador(a) social debe saber aplicar sus conocimientos en la planificación, diseño, implementación, sistematización y evaluación de políticas sociales y poseer habilidades, así como actitudes que les permitan actuar como gestor social activo, reflexivo y crítico, con competencias suficientes para participar en la resolución de necesidades sociales, colocando en práctica los principios teóricosconceptuales, metodológicos y técnicos de la disciplina de TS, que por ser tan diversas las esferas de actuación de estos profesionales, necesitan el desarrollo de estas habilidades en su campo de acción social, tal como se observó en los resultados.

En ese marco de ideas, el $32,1 \%$ de los encuestados expresaron que casi siempre manifiestan las habilidades personales en su acción social, como lo plantean Ramón et al. (2019), sobre todo para vencer las dificultades, teniendo suficiente empuje para llevar a cabo tareas con los cuales se alcanzan los objetivos propuestos pese a las adversidades que se pueden presentar; por tanto, son resilientes para superarlas y continuar con el mismo espíritu de ayuda, sobre todo con quienes son más vulnerables.

Por ello, los resultados indican que el TS demuestra su inteligencia emocional, en cuanto a su autoconocimiento, autoestima, autocontrol, adaptación al cambio, empatía, adecuadas relaciones interpersonales para llevar efectivo trabajo en equipo, resolución de problemas, liderazgo, proactividad; además, habilidades para gestionar recursos, capacidades de información sistémicas para la organización del trabajo, con iniciativa, espíritu emprendedor, entre otros rasgos personales.

\section{Conclusiones}

Los resultados de este estudio, permitieron aportar una información actualizada de cómo estos profesionales de TS asumen sus habilidades para desarrollar su acción social, realizando con ellas sus prácticas en asuntos individuales, grupales y comunitarios; por ello, con esta investigación se determinaron las habilidades del profesional de Trabajo Social en su acción social, pudiendo expresar que en casi todos los ámbitos o áreas 
en las cuales se desenvuelve demuestra sus capacidades en el ser, saber, hacer e interactuar, asumiendo que básicamente lo más importante es como intervenir y mediar, de allí que las habilidades cognitivas o técnicas propician sus conocimientos para actuar, investigar, sistematizar, brindando el apoyo que requieren las personas en sus situaciones bien sean individuales, grupales o comunitarias.

Por lo tanto, se evidenció con los resultados obtenidos que el(la) profesional de Trabajo Social muestra un perfil completo en cuanto a sus habilidades personales, que le permiten ser sensible ante las situaciones que manifiestan las demás personas, entendiéndolos y trabajando con ellos para la solución de problemas y conflictos, para satisfacer necesidades, así como expectativas, tomando en cuenta que son líderes en la comunidad, son personas que por sus capacidades atraen a la gente, brindan confianza y seguridad, lo cual implica tener conocimiento, de allí, la relevancia del saber, para poder actuar con ellos mediante la investigación acción participativa en la elaboración de proyectos que propicien logros individuales, grupales y comunitarios.

En tal sentido, dentro de las cuatro habilidades analizadas, referidas a lo personal, cognitivo, metodológico, social, resulta la comunicación el elemento clave de la acción social, de allí, que es una característica condicionante para lograr el éxito y la efectividad de los proyectos que desarrolle el TS con las personas y las comunidades, debiendo ser asumido durante los procesos de investigación acción participativa, en la intervención, como también en la mediación, y como línea de investigaciones futuras en esta área.

\section{Referencias bibliográficas}

Ander-Egg, E. (1986). Metodología del Trabajo Social. Instituto de Ciencias Sociales Aplicadas.

Arias, A., Arbuatti, A., Giraldez, S. y Zunino, E.
(2013). Secuencias, niveles y procesos: Viejas, y sin embargo presentes, tensiones dentro de la metodología del Trabajo Social. En A. Arias, E. Zunino y S. Garello (Comps.), El proceso metodológico y los modelos de intervención profesional: La impronta de su direccionalidad instrumental y su revisión conceptual actual (pp. 4050). Universidad de Buenos Aires.

Barreto-Pico, M. A. (2017). Abordaje teórico sobre la comunicación y el trabajador social. Dominio de las Ciencias, 3(3), 470-487

De la Rosa, P. (2017). Metodología de la intervención social. Universidad de Valladolid. http://pablodelarosa.sitios. uva.es/sites/pablodelarosa.sitios.uva. es/files/Tema\%20II.pdf

Espinoza, E. E., y Ramón, M. Á. (2020). Competencias del trabajador social egresado de la Universidad Técnica de Machala. Revista Universidad y Sociedad, 12(5), 314-321.

Fantova, F. (2018). Construyendo la intervención social. Papeles del Psicólogo, 39(2), 81-88. https://doi. org/10.23923/pap.psicol2018.2863

Galarza, V. D. (2017). Trabajo social como agente de detección de la dependencia del bono de desarrollo humano por parte de la familia xy del barrio San Luis de Toctiuco en el período octubre 2014 -febrero 2015 [Tesis de pregrado, Universidad Central del Ecuador]. http://www.dspace.uce.edu. ec/handle/25000/10423

García-Domingo, M., y Sotomayor-Morales, E. (2017). El rol del profesional del Trabajo Social en una coyuntura de crisis: Oportunidades de la adaptación a un contexto cambiante. Trabajo Social Global -Global Social Work. Revista de Investigaciones en Intervención social, 7(12), 47-68. https://doi.org/10.30827/tsg-gsw. 


\section{v7i12.5663}

Guillén, J. C., Muñoz, N. B. J., García, M. L., y Giniebra, R. (2021). La mediación: Una estrategia comunicativa para resolver conflictos entre individuos. Encuentros. Revista de Ciencias Humanas, Teoría Social y Pensamiento Crítico, (E-1), 85-99.

Henríquez, A. V. (2017). Plan de acción basado en inteligencia emocional aplicado a la gerencia de aula en los docentes del área de conocimiento pasantia profesional integral del adulto de la facultad de odontología [Tesis de maestría, Universidad de Carabobo]. http://mriuc.bc.uc.edu.ve/ bitstream/handle/123456789/4631/ ahenriquez.pdf?sequence $=1$

Miranda, P., Guerra, L., Calderón, M. P., y Cornejo R. (2020). Conocimientos y habilidades para el Trabajo Social ocupacional: Desafíos para la formación y el ejercicio profesional en esta área. Cuadernos de Trabajo Social, 33(2), 233-245. https://doi. org/10.5209/cuts.64322

Moreno, M., y Molina, N. (2018). La Intervención Social como Objeto de Estudio: Discursos, prácticas, problematizaciones y propuestas. Athenea Digital, 18(3), e-2055. https:// raco.cat/index.php/Athenea/article/ view/v18-n3-moreno-molina

Munuera, P., y Minguela, M. Á. (2019). Mediación comunitaria: Bonding y bridging. Revista Trabajo Social, 1(17), 49-65.

Navarro, F., Almaguer, R., Moreno, F., y Hernández, N. P. (2020). Estrategias comunicacionales en el sector turismo. Revista de Ciencias Sociales (Ve), XXVI(1), 77-90. https://doi. org/10.31876/rcs.v26i1.31312

Novel, G. (2013). El mediador y el manejo de las emociones. Universidad de
Barcelona. https://www.icacor.es/ fileadmin/user upload/archivos/ contenidos/EL MEDIADOR.pdf

Ramón, M. A., Lalangui, J. H., Guachichullca, L. A., y Espinoza, E. E. (2019). Competencias específicas del profesional de trabajo social en el contexto educativo ecuatoriano. Revista Conrado, 15(66), 219-229.

Rodríguez, L. A., Calderón, S. V., y Bravo, J. D. (2019). Retos y limitaciones del trabajador social en las instituciones del distrito $13 \mathrm{~d} 01$ del cantón Portoviejo. Revista Recus, 4(1), 4148. https://doi.org/10.33936/recus. v4i1.1752

Rozas, M. (1998). Una perspectiva teórica metodológica de la intervención en Trabajo Social. Espacio Editorial.

Suárez, N., Pérez, I. C., Rodríguez, A., y Sevilla, S. (2020). Lectura crítica en el desarrollo de habilidades de investigación en profesores de postgrado. Revista de Ciencias Sociales (Ve), XXVI(E-2), 328339. https://doi.org/10.31876/rcs. v26i0.34131

Universidad de Barcelona (2017). Grado de Trabajo Social. Lista completa de competencias. Universitat de Barcelona. https://www.ub.edu/web/ $\mathrm{ub} /$ galeries/documents/estudis/graus competencies/compet g1027 tsocial es.pdf

Universidad Técnica de Manabí (2020). Licenciatura en Trabajador Social. Universidad Técnica de Manabí. https://www.universidades.com. ec/universidad-tecnica-de-manabi/ licenciatura-en-trabajo-social

Vargas-Acosta, E., Ferrer, A. M., García, J., y Rodríguez, I. (2016). Formación profesional del Trabajador Social en Venezuela. Interacción y Perspectiva, 6(1), 78-94. 